\title{
Clinical optical coherence tomography combined with multiphoton tomography of patients with skin diseases
}

\author{
Karsten König ${ }^{1,2}$, Marco Speicher*,1, Rainer Bückle ${ }^{1}$, Julia Reckfort ${ }^{1}$, Gordon McKenzie ${ }^{3}$, \\ Julia Welzel $4^{4}$,Martin J. Koehler ${ }^{5}$, Peter Elsner ${ }^{5}$ and Martin Kaatz ${ }^{5}$ \\ ${ }^{1}$ JenLab GmbH, Schillerstraße 1, 07745 Jena, Germany \\ ${ }^{2}$ Saarland University, Faculty of Mechatronics and Physics, Campus A5.1, 66123 Saarbrücken, Germany \\ ${ }^{3}$ Michelson Diagnostics Ltd., 11A Grays Farm Production Village, Grays Farm Road, Orpington, Kent, BR5 3BD, UK \\ ${ }^{4}$ Dept. of Dermatology and Allergology, Klinikum Augsburg South, 86157 Augsburg, Germany \\ ${ }^{5}$ Dept. of Dermatology and Allergology, University Hospital, Erfurter Straße 35, 07740 Jena, Germany
}

Key words: OCT, multiphoton tomography, skin, SHG, molecular imaging, two-photon, skin cancer, optical biopsy

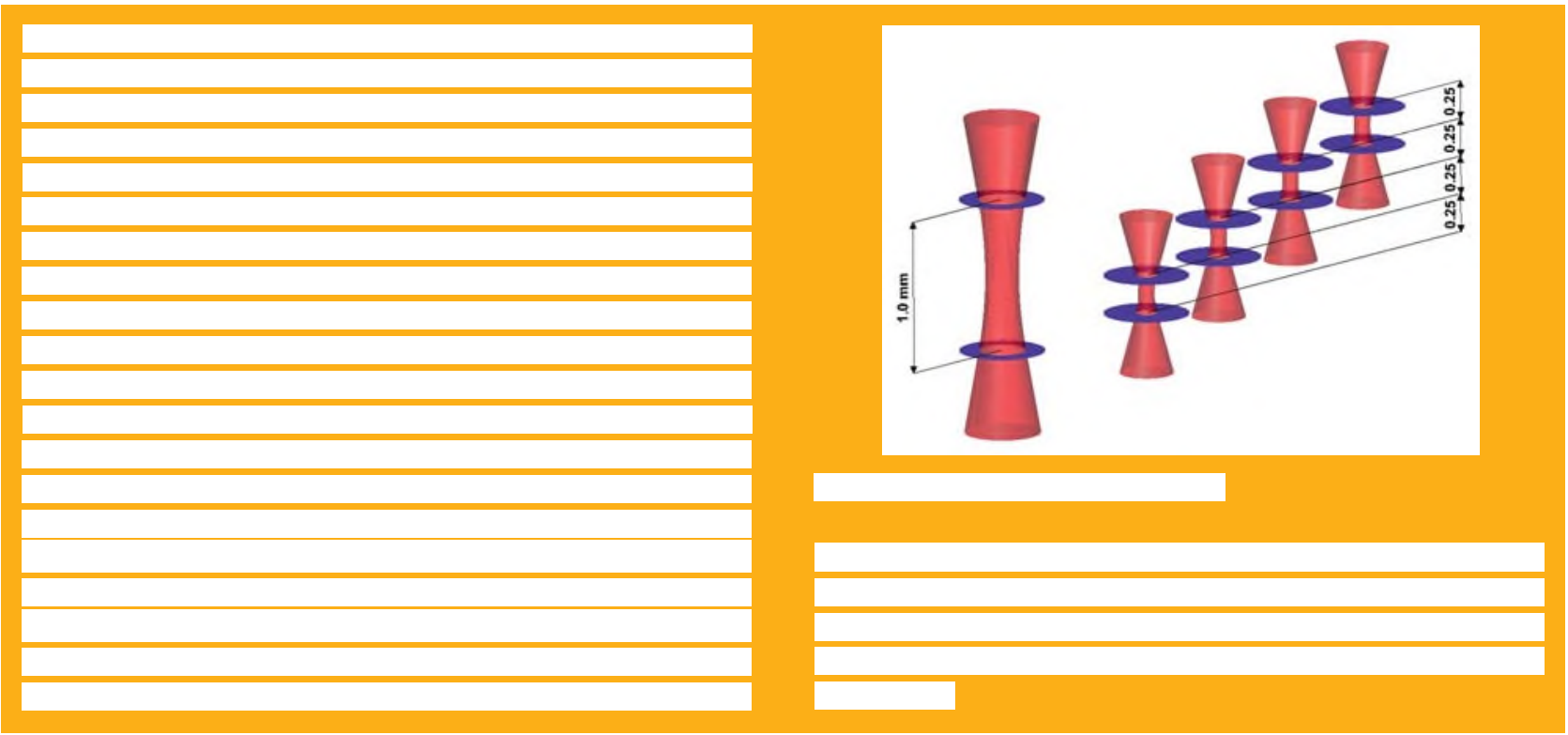

\section{Introduction}

Major tools for non-invasive imaging of skin are ultrasound systems in the frequency range of $7.5 \mathrm{MHz}$ to $100 \mathrm{MHz}$ and optical systems in the ultraviolet (Wood lamp), visible (magnifying glass, dermoscope) and near infrared (NIR) spectral range. NIR systems allow 3D imaging due to the high NIR light penetra- 
tion depth. Major clinical NIR systems include (i) a variety of optical coherence tomographs based on broadband superluminescent diodes (SLD) and tunable/ultrashort lasers, (ii) the confocal reflectance microscope Vivascope (Lucid Inc., Rochester, USA) based on laser diodes, and (iii) the multiphoton tomograph DermaInspect (JenLab GmbH, Jena, Germany) based on a tunable femtosecond $80 \mathrm{MHz}$ titanium:sapphire laser. OCT, reflectance microscopy, and dermoscopy are based on reflected/backscattered light due to intracellular and intratissue modifications of the refractive index. Images can be taken fast within milliseconds at typical powers of few milliwatt of the incident NIR light. In OCT, crosssectional (vertical) images of the tissue such as in ultrasound (B-scan) are obtained by measuring the magnitude and the echo time delay of backscattered light down to a tissue depth of hundreds of micrometers in skin and millimeters in low-scattering ocular tissue. The higher the bandwidth of the light source, the higher the axial resolution. The lateral resolution depends on the laser spot size (beam waist). Time-domain OCT (TD-OCT), spectral domain OCT (SD-OCT, spectral RADAR OCT) and swept-source OCT (SS-OCT) are employed for clinical diagnostics [1]. TD-OCT such as the SkinDex300 (ISIS Optronics $\mathrm{GmbH}$, Mannheim, Germany) requires movement of the reference arm of a Michelson interferometer either by mirror displacement or fiber stretching which limits the OCT speed. SDOCT such as the Callisto from Thorlabs HL AG (Lübeck, Germany) requires a spectrometer to detect the interference modulation. The scan speed can be enhanced by two or three orders of magnitude compared to time-domain systems.

SS-OCT requires a tuneable laser source with a narrow linewidth and fast tuning. This can be achieved by a variety of means, such as using an external-cavity-tuned diode laser, or one of the new generation of frequency domain mode-locking (FDML) lasers with ultrafast tuning capabilities. The fast change (swept) of the laser wavelength can significantly increase the possible speed up to 250000 axial scans per second [1-3].

In SD/SS-OCT, the depth information for the entire depth range of interest is encoded in the spectrum captured. Therefore, the entire depth range must be simultaneously in focus during the spectrum capture. There is a tradeoff between spot diameter and focus depth. Given that OCT signals can be obtained from a maximum skin depth of about $2 \mathrm{~mm}$, a depth of focus of at least $1 \mathrm{~mm}$ is desired. However, this requires focusing optics of low numerical aperture (NA) which limits the minimum beam waist and therefore the lateral resolution to about $20 \mu \mathrm{m}$.

A dynamic focus as applied in TD-OCT is not an option for SD/SS-OCT because data from all depths in the sample are obtained simultaneously. This prob- lem is well known and various methods have been proposed to overcome it. One approach is to use an axicon lens which produces a thin cylindrical 'Bessel beam' [4]. A drawback is that the axicon lens is not suitable for capturing the returning light. When using a different conventional capture lens within a Mach-Zender interferometer, the sensitivity loss can unfortunately exceed $30 \mathrm{~dB}$. A second approach is the use of conventional optics and to employ an $a$ priori knowledge about the beam shape and sample characteristics as a basis for computational extraction of information from out-of-focus regions [5, 6]. The method suffers from high computational overheads and inevitable reduction in sensitivity away from focus.

The multibeam swept source OCT EX1301 (Michelson Diagnostics Ltd., Orpington, UK) utilizes a novel optical set-up involving multiple optical channels which does not suffer from loss of sensitivity or other serious drawbacks [7]. The idea is to partition the depth of field into sub-fields and to provide a separately focused beam for each sub-field. The laser beam in the SS-OCT EX1301 is split into 5 'beamlets' using an etalon-type 'rattle plate' prior to the interferometer beamsplitter. Four of these beams are used to scan the skin and are relayed back to an array of photodiodes where they interfere with four reference beams in the conventional manner. The fifth beam is imaged onto a 5th photodiode to generate a balance signal. OCT systems have been used in dermatology since the 90s mainly for experimental and clinical studies. The morphology and epidermal thickness of normal skin has been investigated [8] as well as bullous diseases [8], psoriasis, contact dermatitis [9], melanocytic lesions [10], and vascular lesions [11]. In contrast to ophthalmology, the technique is not yet applicable for routine diagnostics in dermatology and is still under development. Multiphoton laser scanning tomography [12-20] is based on the non-linear excitation of endogenous fluorophores such as $\mathrm{NAD}(\mathrm{P}) \mathrm{H}$, flavoproteins, keratin, lipofuscin, elastin, collagen, melanin, and metal-free porphyrins as well as on second harmonic generation (SHG) of collagen structures. In principle, single photons can be counted even with picosecond time resolution (time-correlated single photon counting, TCSPC). There is a clinical multiphoton tomograph DermaInspect (JenLab GmbH, Jena, Germany) for high-resolution skin imaging in clinical use in Europe, Asia, and Australia. The typical beam dwell time per pixel is in the range of some microseconds. The uptake of a $512 \times 512$ pixel image $\left(360 \times 360 \mu \mathrm{m}^{2}\right)$ takes $1-8$ s. The incident mean laser power varies between 2 $\mathrm{mW}$ (surface) and $50 \mathrm{~mW}(200 \mu \mathrm{m}$ tissue depth) with transient $\mathrm{kW}$ peak powers. Multiphoton tomographs provide horizontal optical sections of a particular region of interest with submicron resolution down to $200 \mu \mathrm{m}$ tissue depth. They are employed for 
the early detection of melanoma and other skin diseases, to track in vivo sunscreen nanoparticles and pharmacological compounds as well as to evaluate the skin age and the efficiency of anti-aging products. Two-photon microscopy and optical coherence microscopy/tomography have been performed on a variety of samples on a microscope stage [21]. However, until now, no clinical skin imaging study has been conducted based on OCT in combination with multiphoton tomography. Here we report on the first in vivo $\mathrm{OCT} /$ multiphoton optical biopsies of human skin.

\section{Materials and methods}

\subsection{Patients}

The study has been performed on 47 patients (31 males, 16 females, age range: $24-88$ years). They were recruited from the Department of Dermatology, University Jena, in conformity with the Declaration of Helsinki over a period of 8 days. The study was approved by the Ethics Commission of the University Jena. All patients were informed about the procedures and the risks concerning the use of laser devices. They gave written informed consent. Dermatological disorders include epithelial skin cancer (e.g. basal cell carcinoma: 5 cases, squamous cell carcinoma: 4 cases, actinic keratosis: 9 cases), pigmented skin lesions (e.g. melanocytic nevi and malignant melanoma: 11 cases), inflammatory skin disorders (e.g. psoriasis: 2 cases), autoimmune diseases (systemic sclerosis: 2 cases, pemphigus vulgaris: 2 cases), seborrheic keratosis (5 cases), T-cell lymphoma (2 cases), hemangioma ( 2 cases) and tattoos (3 cases). In some cases the diagnosis was confirmed by histopathological examination after surgical excision.

\subsection{OCT systems}

Two OCT systems have been employed for this trial. The swept-source multibeam EX1301 high resolution OCT microscope from Michelson Diagnostics (Orpington, UK) is based on the application of laser beamlets focused to different depths of $0.25 \mathrm{~mm}$ apart from another (Figure 1). The HSL-2000-10 MDL light source (Santec Corporation, Komaki, Japan) with a peak power of $15 \mathrm{~mW}$ at the laser center wavelength of $1305 \mathrm{~nm}$ provides a sweep range of $150 \mathrm{~nm}$ (at $10 \mathrm{kHz})$ that enables real-time monitoring. The laser wavelength scanning speed is in principle controllable up to $25 \mathrm{kHz}$. The acquisition time of a single $5 \times 1.9 \mathrm{~mm}$ scan is approximately $100 \mathrm{~ms}$. The scan width can be enhanced up to $7 \mathrm{~mm}$ [22]. The EX1310 SS-OCT was mounted to a flexible arm

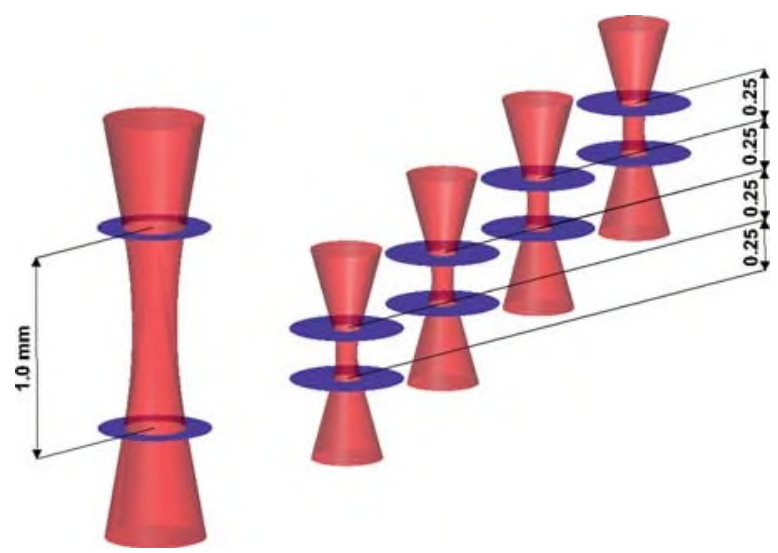

Figure 1 (online color at: www.biophotonics-journal.org) Scheme of the multi-beam OCT. The use of four beams with a total focal range of $4 \times 0.25 \mathrm{~mm}=1 \mathrm{~mm}$ increases the lateral resolution and the contrast compared to onebeam systems.

and modified to be adapted to the skin-tomograph interface used by the multiphoton tomograph DermaInspect. A drop of water was placed on the skin, and the ring and coverslip of the interface were placed on top of the drop and stuck to the skin. Various fluids were tried as index matching agents for the top surface of the cover slip. Eventually, an alcohol spray $(74.1 \%$ Ethanol and 2-Propanol 10\%; Softasept N, Fa. Braun) was observed to give better results than water or indexing matching oil probably because the fine spray gave a very even film approximately 20 microns thick. To allow access to lesions on the torso, the microscope was removed from its mount and held by hand. The ring interface eliminated problems of stand-off and motion and images were easily obtained in vivo. The single beam spectral domain fiber-optic interferometer Callisto (Thorlabs HL AG, Lübeck, Germany) is based on an SLD at a center wavelength of $930 \mathrm{~nm}$, a spectral bandwidth of $50 \mathrm{~nm}$, an A-scan frequency of $1.25 \mathrm{kHz}$, and an output power of $3 \mathrm{~mW}$. A compact handpiece allows any skin region of interest to be reached. The two-dimensional images are recorded with a lateral dimension of $4 \mathrm{~mm}$ to $6 \mathrm{~mm}$.

\subsection{Multiphoton tomograph}

A CE-marked clinical multiphoton tomograph DermaInspect (JenLab GmbH, Jena, Germany) with a flexible mirror arm was employed [17]. The laser source is a tunable $80 \mathrm{MHz}$ femtosecond laser with a tuning range of $690-950 \mathrm{~nm}$, a pulse width of $80 \mathrm{fs}$, and a maximum power of $2 \mathrm{~W}$ (MaiTai, Newport/ Spectra Physics, Mountain View, USA). The laser beam was focused to a submicron spot onto the target by NA 1.3 focusing optics and moved by fast $x, y$ 


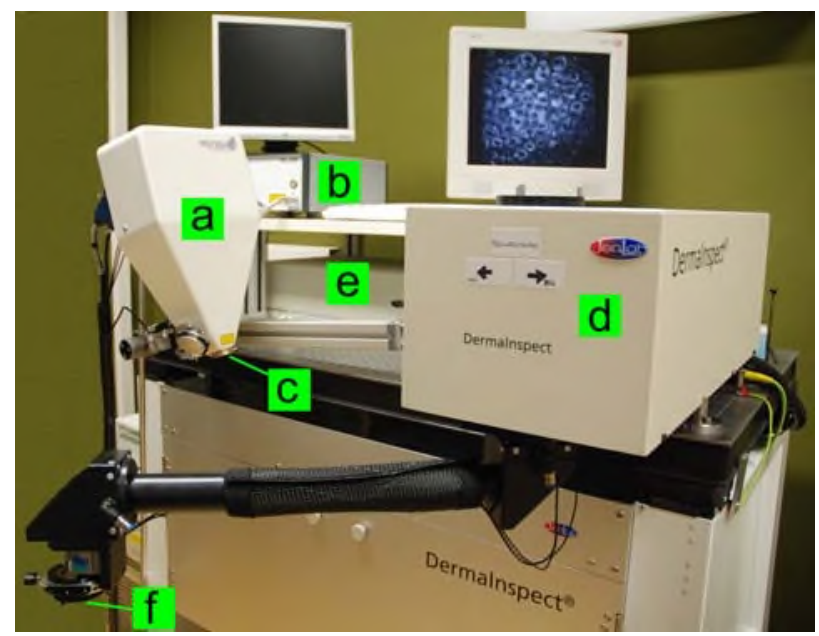

Figure 2 (online color at: www.biophotonics-journal.org) Photograph of the multiphoton tomograph DermaInspect with the multibeam EX1301 SS-OCT system. a: EX1301 SS-OCT; b: laser of the OCT device; c, f: adapter for connection with the metal ring; d: DermaInspect; e: laser of the DermaInspect.

galvoscanners and $z$ piezoactuators. The photomultiplier model H7732 (Hamamatsu Photonics, Hamamatsu City, Japan) and PMTs with a fast response time in the picosecond range served as detectors. Typically, horizontal images were required at a certain focal depth. In addition, "diagonal" images were generated by imaging of sub-fields in different focal depths. A detailed description can be found in reference [12]. Figure 2 represents the multiphoton tomograph DermaInspect combined with the multibeam EX1310 SSOCT system from Michelson Diagnostics.

\subsection{Dermoscopes}

Two dermoscopes from the company Fotofinder Systems (Bad Birnbach, Germany) were employed in the present trial. The video camera Medicam 500 has a resolution of $768 \times 576$ pixels and is additionally equipped with a floodlight. The digital camera Powershot G9 from Canon with a resolution of $4000 \times 3000$ pixels was equipped with white light LEDs based on a combination of a blue LED and phosphor. Fotofinder Mediscope software including the software Moleanalyzer for evaluation of moles via pattern recognition was employed for digital dermoscopy. The Fotofinder software completely controls the cameras and supports different modes, e.g., hands-free portrait, microscopic imaging, body scanning, and polarization imaging. The field of view in microscopic imaging is $11 \times 14 \mathrm{~mm}$. A glass tube with a length of $30 \mathrm{~mm}$ realizes a constant space be-
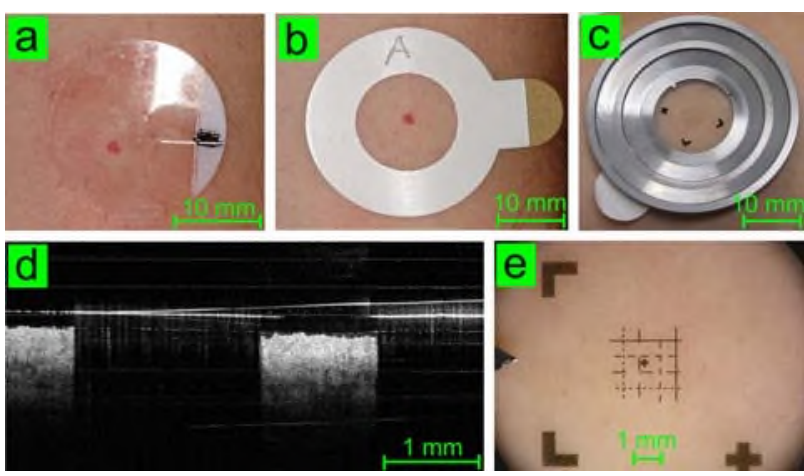

Figure 3 (online color at: www.biophotonics-journal.org) Interface for precise matching of MPT, OCT, and demoscope images consisting of a metal ring with tip, a coverslip with sophisticated marks and a double-faced adhesive foil.

tween the camera and the region of interest on skin. The optic provides magnifications from $20 \times$ to $70 \times$.

\section{Results}

\subsection{Interface for $O C T, M P T$, and dermoscopy}

A big challenge of combining different imaging systems is certainly to match the exact skin structures in the respective images. Different fields of views, special characteristics, and skin interfaces complicate this situation. A special interface consisting of a metal ring and a marked glass coverslip was developed that fits into the DermaInspect as well into the OCT and dermoscopy systems in order to guarantee the measurements of the same skin location of interest. The interface was fixed onto the skin by a special doublefaced adhesive foil. A drop of water was applied between skin and interface. A landmark, in particular for dermoscopy, is a tip in the inner metal ring. This tip and the special marks on the coverslips allow an orientation within the skin disorder. Figure 3 shows the interface. The OCT image (Figure 3e) exhibits signal-poor areas caused by the adhesive foil on top of the skin. The visible segment in the middle of the foil has a length of $1.3 \mathrm{~mm}$. The signal-reach area demonstrates normal skin. When positioned exactly in the center, the size of the OCT skin image corresponds to the inner diameter of the foil.

\subsection{OCT and MPT of healthy normal skin}

The OCT systems provided 2D cross-sectional images similar to the B-scans in ultrasound. Up to 

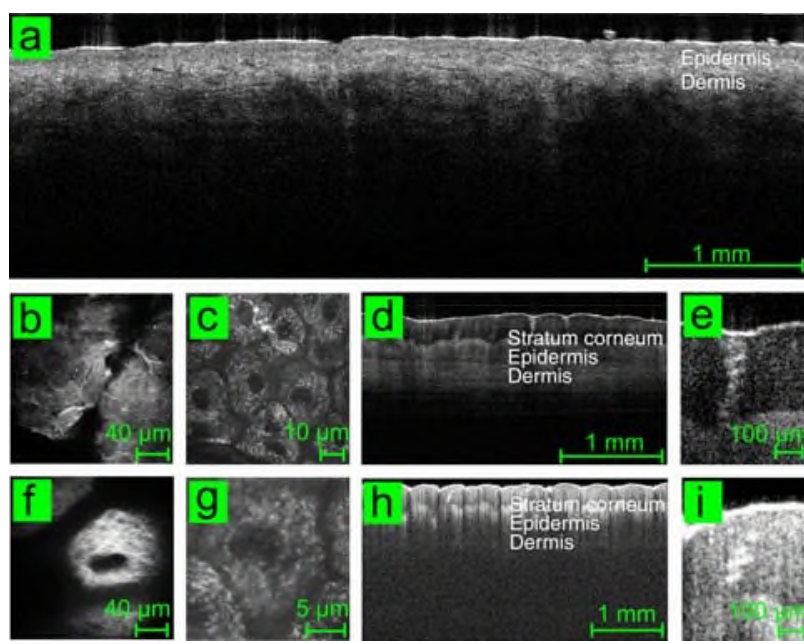

Figure 4 (online color at: www.biophotonics-journal.org) OCT and MPT images of healthy normal skin of a female volunteer. a: OCT image of forearm; $\mathbf{b}, \mathbf{c}, \mathbf{f}, \mathbf{g}$ : multiphoton sections of forearm; d, e: Michelson Diagnostics OCT image of sweat gland(s) in fingertip; h, i: Thorlabs HL OCT image of sweat gland(s) in fingertip.

1200 single A-scans were taken within $1 \mathrm{sec}$ with the Callisto. The swept-source OCT from Michelson Diagnostics provided 10000 A-scans per second. The vertical optical sections (typically $5 \times 1.9 \mathrm{~mm}^{2}$ ) provided information on the architecture of the upper skin like the thickness of the stratum corneum, the thickness of the entire epidermis, the composition of the upper dermis as well as the location of hair follicles and sweat glands (Figure 4). The OCT systems were not able to image single cells or intracellular compartments. Also, no clear differentiation was possible between the different epidermal tissue layers (stratum granulosum, stratum spinosum, stratum basale). In the OCT image, the entrance signal into the skin outshines the stratum corneum. The first homogenously scattering layer is the epidermis (Figure 4d). The dermis possesses several signal-poor structures representing mainly vessels and skin appendages.

The OCT systems were able to provide images with and without the use of the marked $160 \mu \mathrm{m}$ thick coverslip. The adhesive film and the edges of the metal ring with its tip could be detected by OCT (Figure 3). As seen in Figures 4a and d, sufficient signals could be obtained down to a depth of about $1 \mathrm{~mm}$. Sweat glands were the most visible features in OCT images of normal skin at the fingertips where the stratum corneum is very thick (Figures $4 \mathrm{~d}, \mathrm{e}, \mathrm{h}, \mathrm{i}$ ). A diameter and a length of the helix structure of about $100 \mu \mathrm{m}$ and $400 \mu \mathrm{m}$, respectively, of the particular sweat gland (Figure 4e) can be calculated. The multibeam SS-OCT (Figure 4e) provided a better resolution than the single beam SD-OCT system in spite of the higher wavelength (Figure 4i). Multiphoton tomography with the system DermaInspect required the use of a coverslip due to the use of high NA focusing optics. The exposure with intense NIR femtosecond laser pulses resulted mainly in the two-photon excitation of keratin in the outermost layer stratum corneum, of the reduced coenzyme $\mathrm{NAD}(\mathrm{P}) \mathrm{H}$ in the mitochondria in the living cells, of melanin cluster, and of elastin in the upper dermis. $\mathrm{NAD}(\mathrm{P}) \mathrm{H}$ with its maximum of $440-460 \mathrm{~nm}$ provided the major signal in the living cell and enabled imaging of the morphology of single cells and even single intratissue mitochondria (Figure 4g). The non-fluorescent nucleus appeared as dark oval intracellular area. The tissue layers can be distinguished. The optimal excitation wavelength for imaging of the epidermis was found to be between 700 and $800 \mathrm{~nm}$. A good laser wavelength for collagen imaging is between 800 and $950 \mathrm{~nm}$ due to the SHG signal wavelength at half the laser wavelength.

\subsection{Optical biopsies of hemangioma}

OCT images and horizontal as well as diagonal MPT sections of a capillary hemangioma of a 48-year-old male patient have been generated. At first, the hemangioma was studied without the coverslip in order to obtain the exact profile of the lesion (Figure 5a). An altitude of $0.25 \mathrm{~mm}$ of the lesion above the surrounding skin level was determined. The hemangioma appeared as a signal-poor oval, well demarcated structure in the center below a slightly elevated epidermis. A cross section of $0.97 \times 0.51 \mathrm{~mm}^{2}$ (width $\times$ depth) was determined according Figure 5a. Then, the coverslip was placed and fixed with adhesive foil to the skin and the metal ring. Now, OCT as well as MPT with different systems of the same well-defined skin area could be performed. The OCT image of
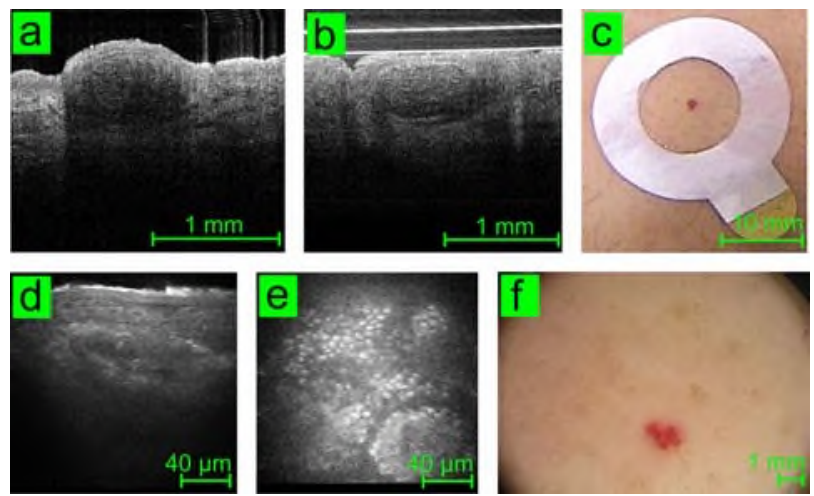

Figure 5 (online color at: www.biophotonics-journal.org) Optical biopsies of a capillary hemangioma. a: OCT image without cover slip; b: OCT image with cover slip on top of the lesion; c, f: dermascopic images; d: diagonal multiphoton tomography slice; e: horizontal multiphoton section of dermal-epidermal junction zone. 
skin covered with the coverslip showed signal-rich features of the glass surface and the glass bottom and provided a length scale (glass thickness: $160 \mu \mathrm{m})$. A slightly different intratissue architecture due to the pressure of the glass is seen. The lumen of the hemangioma calculated from Figure $5 b$ was found to be the same as obtained from Figure 5a. The multiphoton images provided a more detailed view into the architecture of the hemangioma with subvascular and sub-cellular resolution. The $45^{\circ}$ diagonal image (Figure 5d) reflects features seen in the cross-sectional OCT image. MPT cannot image the non-fluorescent erythrocytes but can in principle detect other intravascular cells due to fluorescent mitochondria. The scanning speed in the MPT system is too low to detect moving cells clearly. The one horizontal section (Figure 5e) out of a stack of MPT images shows the junction between the epidermis and the dermis with highly fluorescent basal cells due to the presence of melanin.

\subsection{Optical biopsies of a patient with Pemphigus vulgaris}

A 46-year-old male patient with the diagnosis Pemphigus vulgaris was investigated. Patients with Pemphigus vulgaris suffer from intraepidermal acantholytic blisters which can be easily detected with OCT. Several clefts ("black cavities") can be observed in the image of Figure 6. The star in Figure 6a marks a superficial cleft just below the stratum corneum whereas the arrow points to a signal-poor cavity near the dermoepidermal junction. The cavities are very likely filled with fluid. MPT can also image blisters and epidermal splits (Figure 6b). The image demonstrates less fluorescent signals in the area of clefts likely due to the absence of cell material. In
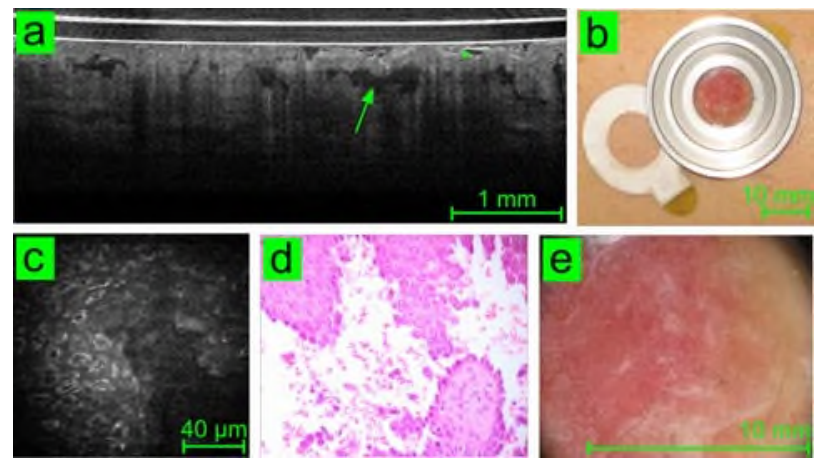

Figure 6 (online color at: www.biophotonics-journal.org) Optical biopsies of a patient with the blister disease Pemphigus vulgaris. a: OCT image with cover slip, subcorneal (*) and subepidermal (arrow) blisters; b, e: dermascopic images; c: MPT section; d: histopathological image.
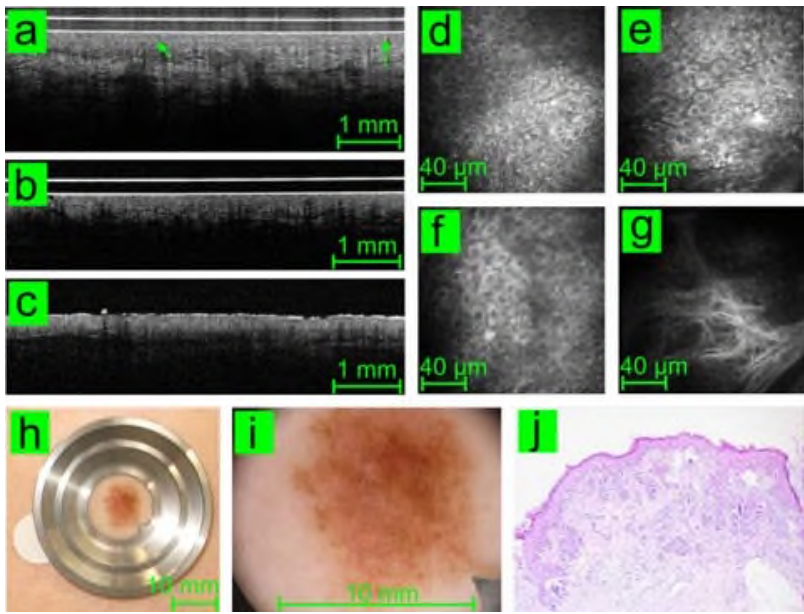

Figure 7 (online color at: www.biophotonics-journal.org) OCT, MPT and dermoscopy images of a 31-year-old female patient with a suspicious pigmented nevus at the left arm. a, b, c: OCT images, highly scattering globules (arrow); d, e, f, g: MPT sections; h, i: dermascopic images; j: histopathological image.

part, surrounding cells exhibited highly fluorescent organelles. The origin of this emission is not clear.

The histopathological image Figure 6e shows an akantholytic blistering and some Tzanck-cells, typical for Pemphigus vulgaris.

\subsection{OCT and MPT imaging of melanocytic lesions}

Figure 7 shows images of a melanocytic lesion of a 31-year-old female patient. In the OCT images, the dermoepidermal junction appeared less well demarcated compared to healthy skin which is probably due to cell aggregates of this lesion. Highly scattering globules (arrows) are visible in this region. MPT provided more precise information. Differences in the layered skin architecture and highly fluorescent melanin clusters can be seen. In part, single dentritic cells were monitored (e.g. Figure 7d, e, f). In the particular region of interest, no infiltration of epidermal cells into the dermis was monitored. The upper dermis exhibited the normal pattern of the extracellular matrix (ECM) network (Figure 7g). At histological examination, the lesion was diagnosed as invasive malignant melanoma (Clark level III, Breslow $0,5 \mathrm{~mm}$ ) with characteristic features such as atypical melanocytes, architectural disarray and melanocytes in upper epidermal layers (Figure 7j). In order to find these criteria as well in MPT, several areas of interest have to be imaged, since due to the irregular architecture of malignant melanoma relevant features may escape evaluation and lead to misdiagnosis. 


\section{Conclusion}

The combination of OCT and MPT provides a unique powerful optical imaging tool for early skin cancer diagnostics and the evaluation of treatments. The skin pathology can be imaged non-invasively in vivo. OCT provides fast wide-field cross-sectional images $\left(5 \times 2 \mathrm{~mm}^{2}\right)$ of the skin area but cannot provide cellular resolution. MPT has superior submicron spatial resolution but is a relatively slow imaging modality with the disadvantage of a small volume of view of about $0.3 \times 0.3 \times 0.2 \mathrm{~mm}^{3}$. The combination combines both (i) the wide-field image with depth information up to $2 \mathrm{~mm}$ and the possibility to identify a particular skin area of interest and (ii) the high-resolution stack of horizontal multiphoton images of this particular region. So far, state-of-the-art technologies have been compared within this first clinical study. Multibeam swept-source OCT has significant advantages regarding resolution and speed compared to time-domain and single beam spectral-domain OCT. Fast FDML lasers will open the way to clinical real-time 3D-OCT in the near future because they are capable to provide hundreds of cross-sectional images within one second [23]. Multiphoton tomographs provide in vivo images with the best resolution. Even single intratissue organelles and single elastin fibers can be imaged. The multiphoton tomograph DermaInspect provides single photon sensitivity and picosecond temporal resolution. This enables imaging of biomolecules with a low fluorescence quantum yield and the detection of backscattered SHG photons. Functional imaging is possible because changes of the redox chain activity due to the detection of the reduced coenzyme $\mathrm{NAD}(\mathrm{P}) \mathrm{H}$ and the dependence of fluorescence lifetime on the microenvironment.

In this study we investigated a variety of skin disorders such as epithelial skin cancer, inflammatory and autoimmune diseases, vascular and pigmented lesions as well as tattoo nanoparticles. In this paper, single cross-sectional OCT images and MPT images out of image stacks of three lesions compared to healthy skin namely hemangioma, Pemphigus vulgaris, and malignant melanoma are presented. OCT provided optical biopsies based on the detection of inhomogeneities such as roundish-shaped or oval signal-poor cavities. The signal-poor regions indicating vascular structures sharply demarcate from the signal-rich microenvironment [11]. The pre-therapeutic knowledge of the lumen and the depth of the vessels can optimize the treatment of hemangiomas and vascular malfunctions. Optimal laser therapy based on selective photothermolysis requires the adjustment of the pulse width to the value of the thermal relaxation time that is a squared function of the vessel diameter [24]. MPT can image vessels only when they occur in the upper dermis within a tissue depth of $0.2 \mathrm{~mm}$. However, hyperkeratosis, atrophy, and the loss of the rete ridge profile in the epidermis which are often accompanied by vascular lesions can be detected with multiphoton biopsies. Bullous diseases such as Pemphigus vulgaris are autoimmune skin diseases in which blisters are formed which can be detected with OCT and MPT. The different groups of bullous diseases can be classified according to the location of the blisters. Pemphigus diseases exhibit signal-poor intraepithelial cavities in OCT and MPT whereas bullous pemphigoid and epidermolysis bullosa acquisita (EBA) lead to subepidermal blister formation. Intraepidermal and dermal blisters occur in burn patients [8]. Within this study, both OCT and MPT provided in vivo information on the pathology regarding the level of blistering and the individual blister architecture including information on intracavity fluids. MPT may also detect impaired adhesion among epidermal cells. Of particular interest is the use of MPT in the case of pigmented lesions such as suspicious nevi. MPT offers the possibility to distinguish between benign nevi and malignant melanoma by detecting single dendritic melanocytes in layers above and beneath the basal cell membrane and further modifications of the epidermal and dermal architecture $[19,20]$. OCT can show infiltration of neoplastic tissue into the dermis and may provide data on the depth of the lesion. These examples of non-invasive OCT/MPT biopsies demonstrate the significant input of this novel 3D imaging technology for the early detection of skin diseases, the potential of pre-operative analysis, and for therapy control. The future development of a single compact 3D OCT/multiphoton system would be a major challenge of current skin imaging technology.

Acknowledgements The authors wish to thank Ms. Nancy Schmidt and Dr. Susanne Biesold from the Dept. of Dermatology at the Friedrich Schiller University Jena and Ms. Vicky Katsoulidou from the JenLab GmbH for the excellent technical assistance as well as the European Commission for financial support within the project FUN-OCT (HEALTHF5-2008-201880). For further support we would like to thank Mr. Marcel Höfer from the JenLab GmbH.

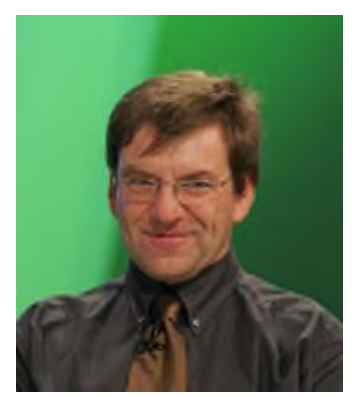

Karsten König studied physics in Rostock, received his diploma, Ph.D. and habilitation degrees at the Friedrich Schiller University Jena, Germany. He worked at the Institute of Laser Technologies in Medicine at Ulm, at the Beckman Laser Institute in Irvine/UCI, at the Institute for Molecular Biotechnology in Jena and at the Medical Faculty of the University Jena. He is currently 
Full professor and Chair of the Department of Microsensors at the Faculty of Mechatronics \& Physics at the Saarland University in Saarbrücken. Prof. König is cofounder of the spin-off company JenLab GmbH. $\mathrm{He}$ is author of more than 200 publications and received a variety of awards.

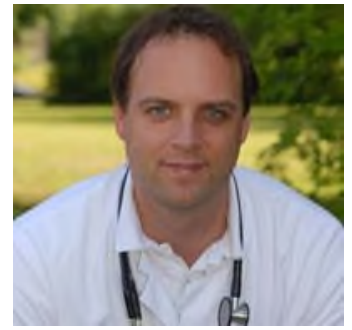

Marco Speicher received a diploma in industrial engineering at the University of Applied Sciences Saarbrücken, Germany in 2003. He worked at SAP Retail Solutions Co. KG, at Fresenius Medical Care $\mathrm{GmbH}$ and at Fraunhofer Institute (IBMT). $\mathrm{He}$ is currently employed at JenLab $\mathrm{GmbH}$, Jena as project manager and studies medicine at the Saarland University. His major interest is skin imaging technologies, especially multiphoton tomography.

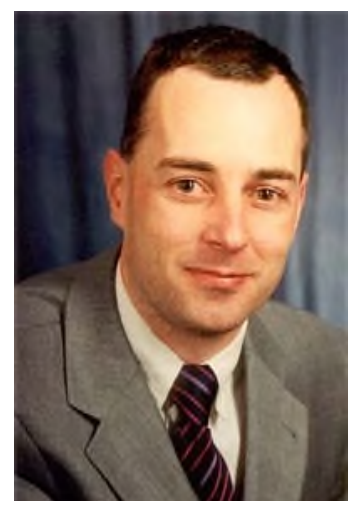

Rainer Bückle graduated as engineer for optical technologies from the University of Applied Sciences Jena $(\mathrm{FH})$ in 1992. He worked at the Fraunhofer Institute (IOF), the Fiedler Optoelektronik $\mathrm{GmbH}$ and the Endoscan $\mathrm{GmbH}$ in Jena. He is currently employed as a senior engineer in the company JenLab $\mathrm{GmbH}$, Jena. His major interest is the development of novel tools for multiphoton microscopy and multiphoton tomography.

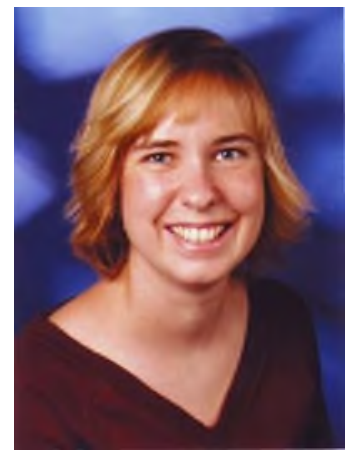

Julia Reckfort started studying "Engineer of Phototechnology" at the Cologne University of Applied Sciences (FHK) in 2004. After an internship at the Fraunhofer Institute in Duisburg (IMS) she decided to specialize on medical imaging technologies. In 2007 she studied for two semesters at the RMIT University, Melbourne, Australia. She is going to finish her thesis about multiphoton tomography, confocal microscopy and dermoscopy supervised by Professor Stefan Altmeyer (FHK) and Professor Karsten König (Saarland University, JenLab) in April 2009.

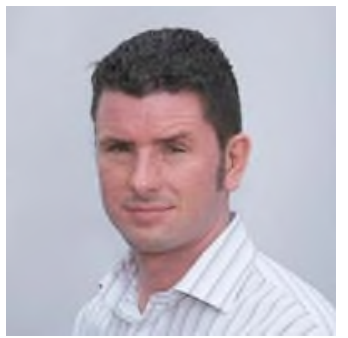

Gordon McKenzie has ten years experience in the field of biomedical optics, including a Ph.D. in the interaction of laser light with tissue under Prof. BryanstonCross. He has established an extensive network of senior clinical and health sector contacts, and is an expert at working in the interface between clinicians and technologists. His key role is to ensure that OCT products of Michelson Diagnostics Ltd. are developed in close collaboration with the clinical community, effectively addressing the problems posed by cancer diagnosis and treatment.

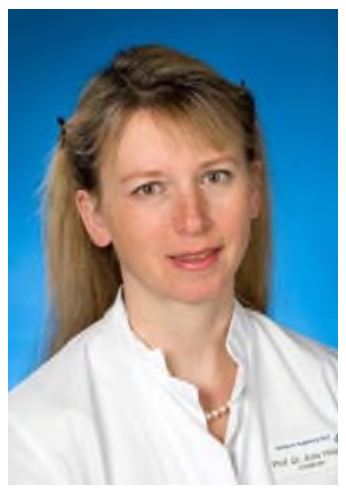

Julia Welzel studied medicine in Hamburg and Lübeck, Germany. She received the M.D. and habilitation degree at the University of Lübeck. Since 1994 she is working as principal investigator of several clinical and experimental studies (phase III and IV: basal cell carcinoma, melanoma, acne, skin ageing, wound healing). She performed several trainings in ICH-GCP (last in September 2008). She is the head of the Department of Dermatology and Allergology of the General Hospital Augsburg, Germany and published more than 47 peer reviewed papers.

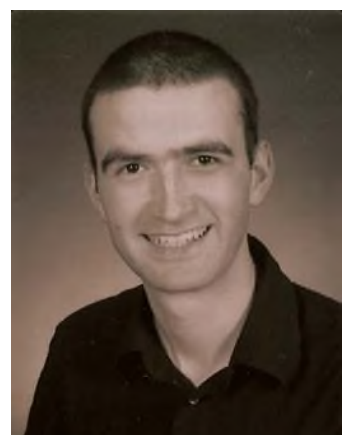

Martin Johannes Köhler studied medicine in Jena, Leipzig, Salamanca (ES), Barcelona (ES) and Toulouse (F) and wrote in 2006 his doctoral thesis on "Melanoma cell-induced expression of proteases and cytokines in dermal fibroblasts". Currently he works at the Department of Dermatology, Jena University, Germany. 


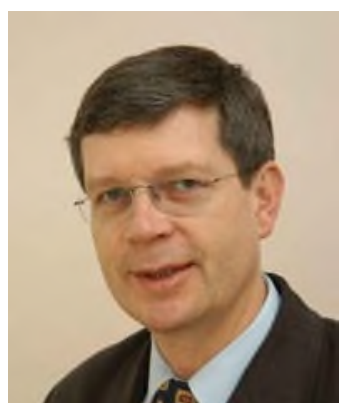

Peter Elsner studied medicine and received the M.D. and habilitation degrees at the Julius-Maximilians-University in Würzburg Germany. He worked at the Department of Dermatology at UCSF in San Francisco, as private lecturer at the University in Würzburg and at the University in Zurich, Switzerland before he became Full Professor and Director of the Department of Dermatology at the Friedrich Schiller University Jena. Prof. Elsner is author of more than 300 scientific publications and 30 books.

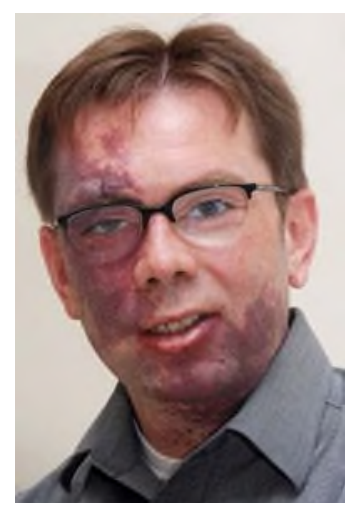

Martin Kaatz studied medicine and received his M.D. degree at the Friedrich Schiller University Jena. He works as the senior physician dermatologist at the Department of Dermatology and Allergology in Jena. His research interests are in the fields of allergology, wound healing, and oncology. Dr. Kaatz is involved in more than 20 clinical trials (phase II-IV) in particular in the field of the therapy of malignant melanoma.

\section{References}

[1] W. Drexler and J. G. Fujimoto, Optical Coherence Tomography. Springer-Verlag GmbH, Heidelberg (2008).

[2] A. F. Fercher, W. Drexler, C. K. Hitzenberger and T. Lasser, Rep. Prog. Phys. 66, 239-303 (2003).

[3] V. J. Srinivasan, D. C. Adler, Y. Chen, I. Gorczynska, R. Huber, J. S. Duker, J. S. Schuman, and J. G. Fujimoto. Invest. Ophth. Vis. Sci. 49, 5103-5110 (2008)

[4] K. S. Lee, C. Koehler, E. G. Johnson, and J. P. Rolland, SPIE Proceed 6079, 607919-607918 (2006).
[5] D. L. Marks, T. S. Ralston, P. S. Carney, and S. A. Boppart, SPIE Procced 6429, 64291R 64299 (2007).

[6] T. S. Ralston, D. L. Marks, P. S. Carney, and S. A. Boppart, SPIE Proceed 6446, 644608-644611 (2007).

[7] J. Holmes, S. Hattersley, N. Stone, F. Bazant-Hegemark, and H. Barr, SPIE Proceed 6847, 68470O (2008).

[8] M. Mogensen, H. A. Morsy, B. M. Nurnberg, and G. B. Jemec, JEADV 22(12), 1458-1464 (2008).

[9] J. Welzel, M. Bruhns, and H. H. Wolff, Arch. Dermatol. Res. 295, 50 (2003).

[10] V. de Giorgi, M. Stante, D. Massi, L. Mavilia, P. Cappugi, and P. Carli, Exp Dermatol. 14(1), 56-59 (2005).

[11] C. Salvini, D. Massi, A. Cappetti, M. Stante, P. Cappugi, P. Fabbri, and P. Carli, Skin Res Technol 14(1), 89-92 (2008).

[12] K. König and I. Riemann. J. Biomed. Optics 8, 450459 (2003).

[13] K. König, A. Ehlers, F. Stracke, and I. Riemann. Skin Pharmacol. Physiol. 19, 78-88 (2006).

[14] K. König, A. Ehlers, I. Riemann, S. Schenkl, R. Bückle, and M. Kaatz, Microsc. Res. Techniq. 70, 398-402 (2007).

[15] M. J. Koehler, K. König, P. Elsner, R. Bückle, and M. Kaatz, Opt. Lett. 31, 2879-81 (2006).

[16] K. König, J. Biophoton. 1, 13-23 (2008).

[17] K. König, M. Weinigel, D. Hoppert, H. Schubert, M. J. Köhler, M. Kaatz, and P. Elsner, J. Biophoton. 1, 506-513 (2008).

[18] M. J. Koehler, S. Hahn, A. Preller, P. Elsner, M. Ziemer, A. Bauer, K. König, R. Bückle, J. W. Fluhr, and M. Kaatz, Exp. Dermatol. 17, 519-523 (2008).

[19] E. Dimitrow, I. Riemann, A. Ehlers, M. J. Koehler, J. Norgauer, P. Elsner, K. König, and M. Kaatz, Exp. Dermatol. DOI:10.1111/j.1600-0625.2008.00815.x (2009).

[20] E. Dimitrow, M. Kaatz, M. Ziemer, J. Koehler, J. Norgauer, and K. König, J. Invest. Dermatol. 129(7), 1752-1758 (2009).

[21] S. Tang, T. B. Krasieva, Z. Chen, and B. J. Tromberg, J. Biomed. Opt. 11, 020502-1-3 (2006).

[22] P. H. Tomlins, P. Wooliams, M. Tedaldi, A. Beaumont, and C. Hart, SPIE Proceed. 6847, 68472Q-68478 (2008).

[23] B. R. Biedermann, W. Wieser, C. M. Eigenwillig, T. Klein, and R. Huber, Opt. Express 17(12), 99479961 (2009).

[24] R. R. Anderson and J. A. Parrish, Science 220(4596), 524-527 (1987). 